\title{
Application of advanced edge diagnostics for transport studies in the stochastic boundary of TEXTOR-DED
}

\author{
O. Schmitz* , S. Brezinsek*, I. Classen ${ }^{\dagger}$, M.Clever*, J.W. Coenen*, E. Delabie ${ }^{\dagger}$, \\ K.H. Finken*, M.W. Jakubowski**, M.Kantor*, A. Krämer-Flecken*, U. Kruezi*, \\ M. Lehnen*, Y. Liang*, D. Reiter*, U. Samm*, B. Schweer*, G.W.Spakman ${ }^{\dagger}$, \\ H. Stoschus*, G. Telesca*, B. Unterberg*, E. Uzgel*, Y.Xu* and the TEXTOR team*
}

\author{
*Institut für Energieforschung 4 - Plasmaphysik, Forschungszentrum Jülich GmbH in der \\ Helmholtzgemeinschaft, Trilateral Euregio Cluster, Association Euratom-FZJ, D-52425 Jülich, Germany \\ ${ }^{\dagger}$ FOM-Institute for Plasma Physics Rijnhuizen, Trilateral Euregio Cluster, Association EURATOM-FOM, \\ PO-Box 1207, 3430 BE Nieuwegein, The Netherlands \\ **Max-Planck-Institut für Plasmaphysik, IPP-EURATOM Association, Greifswald, Germany \\ ${ }^{\ddagger}$ Laboratory for Plasma Physics, Association EURATOM -Belgian State, KMS - ERM, \\ Trilateral Euregio Cluster, B-1000 Brussels, Belgium
}

\begin{abstract}
In this paper the edge diagnostic equipment at the TEXTOR tokamak is described and its importance for detailed transport studies in different shapes of perturbed plasma edge layers is demonstrated. At TEXTOR an external resonant magnetic perturbation is induced by the dynamic ergodic divertor DED and various topologies of the perturbed magnetic field are feasible. A helical divertor topology, dominating magnetic island chains or an ergodicity dominated layer can be evoked adapting the perturbation field base mode number, the perturbation field strength and the plasma equilibrium. As unique feature the DED perturbation field can be rotated with frequencies of up to $10 \mathrm{kHz}$. The impact of the very different magnetic field structures and the fast rotation of the external perturbation field on local and global transport properties is analyzed here on selected examples from the various edge diagnostics with high time and / or high spatial resolution. Based on this basic knowledge the perturbed edge layer was adapted to control the particle exhaust characteristics and the impurity transport. Both, an improvement in the particle confinement was found as well as a controlled degradation - the so called particle pump out effect - correlated to a pronounced screening of impurities.
\end{abstract}

PACS: $28.52 .-\mathrm{s}, 52.25 . \mathrm{Fi}, 52.40 . \mathrm{Hf}, 52.55 . \mathrm{Dy}, 52.55 . \mathrm{Rk}, 52.70 . \mathrm{Gw}, 52.70 . \mathrm{Kz}, 52.70 . \mathrm{Nc}$

\section{INTRODUCTION}

The experimental detection of three dimensional plasma structures and the analysis of correlated transport characteristics require advanced edge diagnostic equipment. This is relevant especially for the understanding of edge transport in three dimensional magnetic field topologies existing in stellarators as well as in tokamak plasmas with non axissymetric, resonant magnetic perturbations (RMP). Here RMPs are a modern objective of research as a tool to mitigate the harmful energy bursts caused by edge localized modes (ELMs) in H-mode plasmas with improved particle and energy confinement $[1,2,3]$. As this technique is a promising candidate for the ITER tokamak experiment the underlying mechanisms have to be explored. In stellarators transport analysis is needed for the understanding of the two major exhaust concepts [4]. The transport around magnetic islands is of importance for the development of the island divertor concept [5] and also understanding the mechanisms in helical structures which inherently exist in the stellarator symmetry are required for the exploration of the helical divertor [6]. The in detail analysis of transport phenomena in these kind of magnetic topologies contributes to the assessment of the potential of these exhaust concepts and the mechanisms behind ELM mitigation by RMP application.

At the TEXTOR tokamak the Dynamic Ergodic Divertor (DED) was designed as a flexible setup to investigate the generic features of plasma boundaries with perturbed magnetic field structures [7]. As depicted in figure 1 it consists of a set of $16+2$ coils implemented into the vacuum vessel and winding helically around the torus at the high field side (HFS). They create a perturbation spectrum with main resonances around the $q=3$ surface and poloidal/toroidal base mode numbers of $m / n=3 / 1,6 / 2,12 / 4$ can be selected by different wiring of the perturbation coils. The fields induced can be rotated with high frequencies of $v=1-10 \mathrm{kHz}$. To facilitate the experimental investigation 

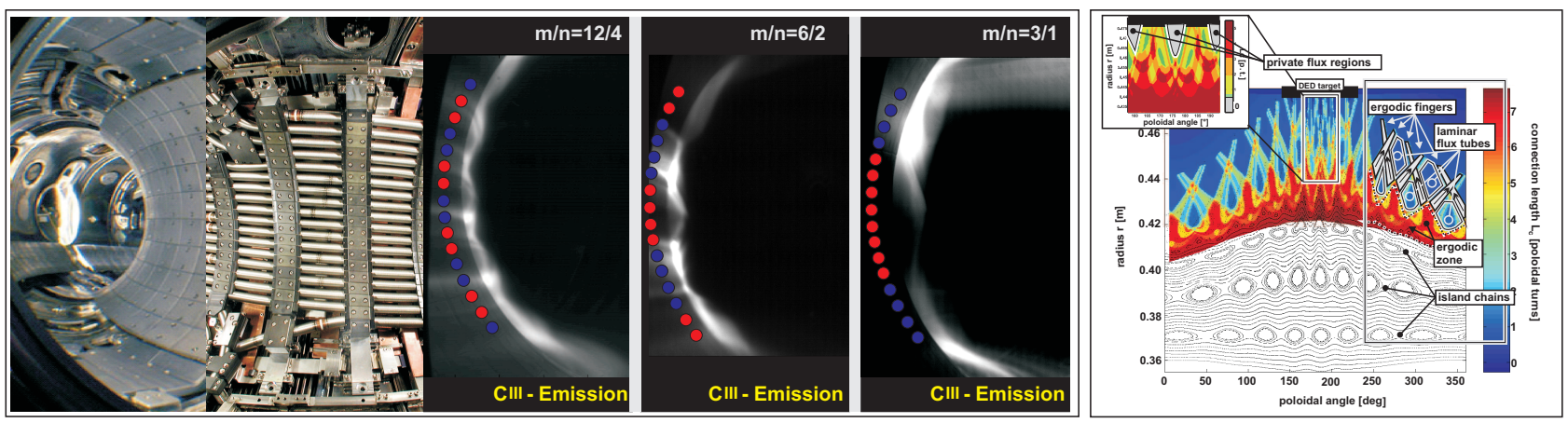

FIGURE 1. Setup of the Dynamic Ergodic Divertor (DED). From left: tangential view into the vacuum vessel with DED target, DED coil set without coverage and impression of the DED induced edge structure in CIII light for all three base mode configurations with comparable perturbation current of $\approx 0.7 \times I_{D E D \text { max }}$. The figure on the right shows and example of the rangy magnetic field topology in $m / n=12 / 4$ base mode for a comparable plasma discharge as shown in the CIII emission pictures. Shown here is a combination of a Laminar Plot and a Poincaré Plot. The file line's connection lengthes are color coded in units of poloidal turns.

the magnetic topology induced can be moved by low frequency $(v=1,2 \mathrm{~Hz})$ strike point sweeping. The right part of figure 1 shows the perturbed magnetic topology as example for the challenging diagnostic needs to resolve the transport characteristics in such a rangy and fully 3D helical magnetic field structure. Here field lines with short and long connection lengths $L_{c}$ to the DED target can be distinguished and therefore very different transport characteristics were found $[8,9,10,11,12,13,14]$. Field lines with short $L_{c}$ are bundeled in the so called laminar flux tubes and they act as a multipolar helical scrape off layer (SOL), dominated by parallel transport along the field lines to the wall elements. In contrast, the field lines with long $L_{c}$ - called ergodic field lines - show increasing field line diffusion and they therefore generate an stochastic domain with enhanced radial transport. Both domains are poloidally interwoven and their radial and poloidal extensions can be varied from a few millimeters to up to several centimeters by changing the base mode configuration [15]. However, beyond this standard stochastic layer the perturbation level on the different resonant layers can be adapted such that either magnetic islands are dominating for the edge transport properties - and the global confinement - or these islands can be destroyed leading to a scenario where the long, ergodic field lines with high diffusivity and therefore prevailing radial transport are dominating.

In this paper the importance of good edge diagnostic equipment for the resolution of the transport properties in these very different topologies will be discussed based on experimental results from various diagnostic techniques applied at different toroidal and poloidal locations. These basic findings allow to gain dedicated control on the particle and heat exhaust and the application of the basic understanding will be shown exemplarily on two regimes discovered: an improved particle confinement and in contrast a controlled degradation of the particle confinement - the so called particle pump out.

\section{DIAGNOSTIC SUITE APPLIED}

According to the needs for comprehensive edge characterization TEXTOR was equipped with a efficient set of edge diagnostic systems of which some are unique. This suite available and applied for the transport analysis is described here. In figure 2 an overview on the various systems is given and their toroidal and poloidal location is sketched. Table 1 gives an commented overview with the parameters obtained and the system's specifications. This overview shows that crucial parameters for edge transport analysis can be obtained at various toroidal and poloidal location with high spatial and / or high time resolution. Three systems or experimental techniques, respectively will be outlined here and their performance will be demonstrated in the next section on related results describing the basic transport features of the DED induced stochastic layer. However, this is an exemplarily selection only and an finalizing overview of the manifold edge diagnostics is given in table 1 and the many more results obtained are referenced in the conclusion.

- The technique of beam emission spectroscopy (BES) on thermal atomic He beams was established at TEXTOR as reliable method for measuring electron density $n_{e}(r, t)$ and temperature $T_{e}(r, t)[16,17]$. The possibility to measure $n_{e}$ and $T_{e}$ with BES on He stems from the different temperature and density dependence of the population densities 


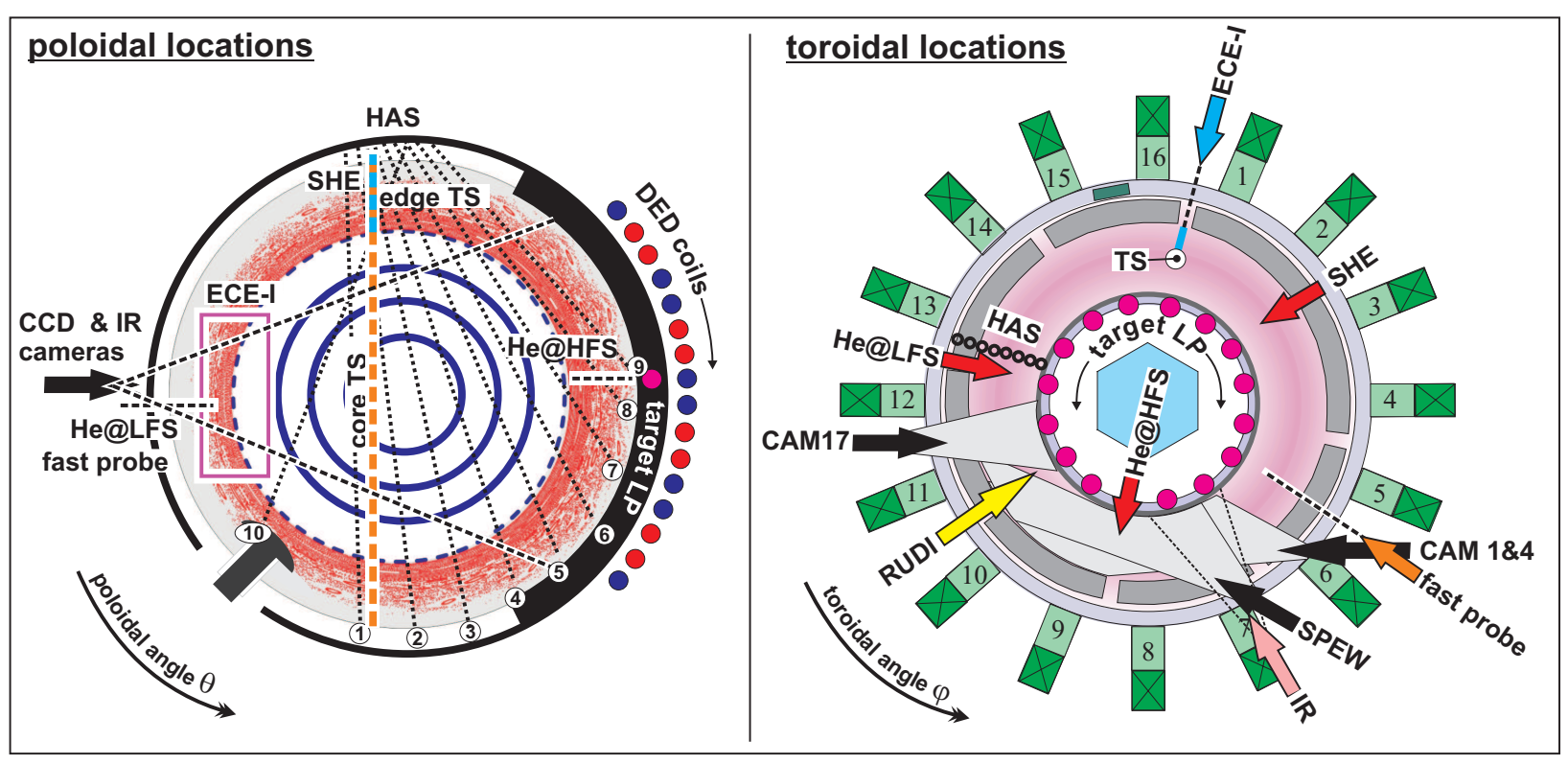

FIGURE 2. Overview of the edge diagnostic equipment used for the transport characterization and analysis in the stochastic edge layer of TEXTOR-DED. The specifications of the systems and the parameters measurable are listed in table 1

$n_{i}\left(n_{e}, T_{e}\right)$ of the He energy levels in both spin systems. For the triplet states $n_{i}\left(n_{e}, T_{e}\right)$ has its maximum at lower values of $T_{e}$ than $n_{i}\left(n_{e}, T_{e}\right)$ in the singlet system. The radial profile of the ratios $R_{1}(r, t)=I_{1}(r, t) / I_{3}(r, t)$ from two specific line intensity profiles $I_{1}(r, t)$ and $I_{3}(r, t)$ in both systems allow therefore to deduce $T_{e}(r, t)$. In order to measure $n_{e}(r, t)$ the different density dependence of specific $n_{i}\left(n_{e}, T_{e}\right)$ within the singlet system is utilized and the ratio $R_{2}(r, t)=I_{3}(r, t) / I_{2}(r, t)$ of two measured line intensity profiles $I_{2}(r, t)$ and $I_{3}(r, t)$ from this spin system let us deduce $n_{e}(r, t)$. We perform these measurements at the low field side (LFS) and the high field side (HFS) of the TEXTOR vessel in injecting a well defined amount of He through a collimating nozzle structure consisting of a set of 340 micro tubes with a diameter of $d_{t}=270 \mu \mathrm{m}$ each. This arrangement provides for typical pressures of $p_{v}=4-10 \mathrm{mbar}$ in the reservoir an effusive, thermal beam source for these two beam systems $[18,19]$. The resulting He beams have a divergence of $\Delta \xi= \pm 20 \mathrm{deg}$ and for a source temperature of $T_{S}=450 \mathrm{~K}$ a mean averaged effusive beam velocity of $v_{E}=1.5 \mathrm{~km} / \mathrm{s}$ resulting in a averaged local He density in the beam of $n_{H e}=5.0 \times 10^{11} \mathrm{~cm}^{-3}$. The line emission is observed with a view perpendicular to the thermal atomic beam to get the best spatial resolution possible. The time resolution of the observation systems allow $\Delta t>2 \mathrm{~ms}$ for the LFS system and $\Delta t>20 \mathrm{~ms}$ for the HFS system. From the emitted He light three suitable line emissions are extracted by 2-cavity interference filters with a spectral resolution of $\Delta \lambda_{F W H M}=1.5 \mathrm{~nm}$ at LFS or with a high resolution spectrometer at the HFS, respectively. For observation we choose for the singlet states the line intensity of the transition $3{ }^{1} \mathrm{D} \rightarrow 2{ }^{1} \mathrm{P}$ at $\lambda_{1}=667.8 \mathrm{~nm}\left(I_{1}(r, t)\right)$ and $3{ }^{1} S \rightarrow 2{ }^{1} P$ at $\lambda_{2}=728.1 \mathrm{~nm} I_{3}(r, t)$. The ratio of these two lines is mainly determined by $n_{e}$. To deduce $T_{e}$ we use the ratio of the triplet transition $3^{3} S-3^{3} P$ at $\lambda_{3}=706.5 \mathrm{~nm}$ as $I_{2}(r, t)$ and the singlet transition $2^{1} P-3^{1} S$ at $\lambda_{2}=728.1 \mathrm{~nm}$ as $I_{3}(r, t)$. The advantages of these lines are a good line intensity in the range of the target plasma parameters and the fact that they are experimentally easy accessible as these transitions emit in the visible range of the spectrum. The measured line ratios $R_{1}(r, t)$ and $R_{2}(r, t)$ are compared to the ones modeled with a collisional radiative model (CRM). Here a stationary model is used for the direct experimental evaluation assuming that the population densities in the energy level system modeled are in a temporal equilibrium. This assumption is justified for the observation of quasi-stationary events on the $10-100 \mathrm{~ms}$ time scale. For faster events it need to be considered and defines a boundary for the time resolution with this method as discussed below.

The capability of this well established diagnostic technique was recently extended by a new super-sonic Helium beam (SHE) system. Here the collimating nozzle structure and its effusive thermal source characteristics is replaced by a more sophisticated injection system [20]. In this principle the He is injected from a high pressure reservoir $\left(P_{H e}=70-100\right.$ bar $)$ towards a specially designed nozzle-skimmer system. The skimmer strips out a small collimated He beam which is penetrating into the plasma. The beam exhibits super-sonic behavior due to the expansion from the 
high pressure reservoir behind the skimmer into the low pressure, vacuum environment of the plasma chamber. It has a low beam divergence of $\Delta \xi<5 \mathrm{deg}$ with a beam width of $\Delta w_{F W H M} \leq 12 \mathrm{~mm}$ and therefore a local atomic density of $n_{H e}=5 \times 10^{11} \mathrm{~cm}^{-3}$ in the beam is provided with a absolute He flow of $\Phi_{H e}=1-5 \times 10^{17} \mathrm{He}$ atoms $s^{-1}$. This system recently mounted at TEXTOR [20] equipped with an highly light sensitive observation system. It consists of three photo-multiplier tubes (PMT) (one for each wave length, spectral separation is done with interference filters also) each with 32 channels with anode area of $A_{a n}=0.8 \times 7 \mathrm{~mm}^{2}$ leading to a spatial resolution in the measurement of $\Delta r=2 \mathrm{~mm}$. The analogous signals received with this advanced observation system are digitized by a 24 channel data acquisition (DAQ) system with a sampling frequency of $v_{s}=800 \mathrm{kHz}$ and $14 \mathrm{bit}$ digital resolution. The SHE beam is operated in pulsed mode with $\tau_{P}=120 \mathrm{~ms}$ pulse length and $420 \mathrm{~ms}$ interruption for background substraction and pumping of the skimmer volume. Between each pulse the system multiplexes to the 8 out of the 32 PMT channels of each spectral channel. After four He pulses the measurement is at the initial position again and a radial range of $6.2 \mathrm{~cm}$ can be scanned with this system. Concerning the time resolution the relaxation processes in the He population densities are the limiting factor. Depending on the local plasma electron density the energy levels chosen for observation and modeling need different time scales to come to an equilibrium in the population density. This time constant - the relaxation time $\tau_{r}$ - is longer for the metastable triplet state used, accounting in the range of $0.2 \mu s<\tau_{r}<5 \mu s$ for $0.1 \times 10^{18} \mathrm{~m}^{-3}<n_{e}<20.0 \times 10^{18} \mathrm{~m}^{-3}$. Testing the stationary evaluation method on time fluctuating sinusoidal signals showed that for typical edge densities at a radial position around the last closed flux surface (LCFS) the effective time resolution is $\Delta t=5-10 \mu \mathrm{s}$ leading to a frequency resolution of $\Delta v=100-200 \mathrm{kHz}$ [18]. Fast events in this time scale can be resolved and first measurements from DED operation in $1 \mathrm{kHz} \mathrm{AC}$ mode will be presented and discussed later.

- For fast, two-dimensional measurements of the electron cyclotron emission (ECE) a 128 channel ECE radiometer is mounted at TEXTOR operated by the FOM-Institute for Plasma Physics in Rijnhuizen, the Netherlands [21, 22, 38]. ECE radiation from different vertical positions in the plasma is imaged on 16 receivers. Each receiver has 8 frequency channels allowing for 2D measurements of $T_{e}(r, \vartheta, t)$ in the poloidal $\vartheta$ and radial $r$ coordinate. Each vertical $T_{e}$ slice of the plasma is measured with 16 poloidal and 8 radial channels with a frequency resolution of up to $\Delta v \simeq 500 \mathrm{kHz}$. The radial location of this measurement slice depends on the magnetic field and for the system described here the receiver frequency is tunable in a wide range from 85 to $130 \mathrm{GHz}$. By that the radial location of the measurement volume can be adapted for a broad range of settings for the common magnetic field scales of TEXTOR. For the investigations described here the observation volume was set to the plasma edge layer in between $0.75<r / a<1.0$ ( $r$ is the plasma radius, $a$ the minor radius as distance to the LCFS). An example for the extraordinary capability provided by this system will be given in the next section.

As these measurements were performed at the LFS of the TEXTOR tokamak the measurement capability in front of the DED target is more limited. In principle the ECE-I channels can be tuned to be located at HFS and as mentioned a thermal atomic He beam is mounted there measuring $n_{e}(r, t)$ and $T_{e}(r, t)$ profiles right in front of the DED target. However, as this one localized measurement in the complex 3D helical structure induced, measurements along the toroidal coordinate are desired in particular in front and on the DED target. Here CCD cameras equipped with interference filters are useful to get an impression on the particle fluxes and the particle source location while the fast framing IR camera is capable to measure the heat flux distribution. The application of Langmuir probes is in particular promising as they are well designed to measure crucial parameters in the divertor downstream region on the target.

- Therefore 16 Langmuir probes (LP) are mounted in the DED target tiles. These are dome probes sticking out by $2 \mathrm{~mm}$ of the tile surface. They are located at the poloidal angle $\vartheta=185 \mathrm{deg}$, i.e. slightly above the equatorial midplane and evenly distributed along the toroidal angle. These probes measure the ion saturation current $I_{\text {sat io }}(t)$, the electron temperature $T_{e}(t)$ and the floating potential $V_{\text {float }}(t)$. The plasma potential $\Phi_{P}(t)$ and the electron density $n_{e}(t)$ can be evaluated from these measurements. The probes mounted at TEXTOR are commonly operated in voltage sweep mode with $200 \mathrm{kHz}$ frequency resolution. In the following the analysis of the helical divertor properties with the set of diagnostics at LFS and this toroidal probe array will be described. 


\section{EXAMPLES FOR TRANSPORT STUDIES WITH THESE DIAGNOSTICS}

As shown in figure 1 the plasma edge layer is strongly influenced by the DED induced resonant magnetic perturbation. In the close proximity of the DED target - right in front of the perturbation source - the magnetic topology guides particles and energy in the ergodic finger structure towards the DED target [12, 23]. In between these fingers a private flux region exists with a cold and diluted plasma [13]. Therefore the particles released from the wall penetrate through this domain getting ionized in the ergodic finger structure and the deeper stochastic boundary. Its structure for all three base modes is visualized in figure 1 as $C I I I$ emission. On the LFS of the torus we expect interleaved ergodic and laminar domains as shown in the magnetic topology (right plot in figure 1). The ECE-I system is capable to resolve the electron temperature reaction on these interwoven transport domains. Figure 3 shows an example for this analysis in close correlation to the topology of the perturbed magnetic field. A set of two discharges was analyzed here, one with standard settings for plasma current $I_{P}$ and toroidal magnetic field $B_{t}$ and one discharge where $I_{P}$ and $B_{t}$ were reversed.

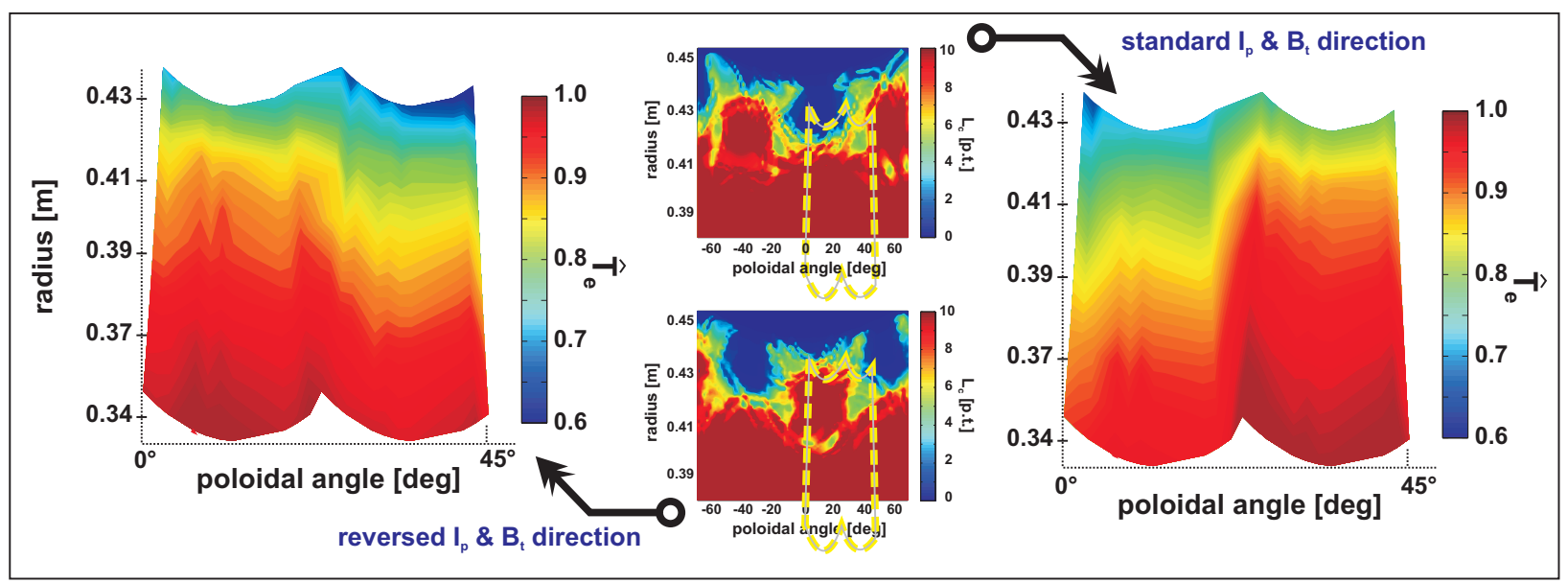

FIGURE 3. Normalized change of the electron temperature change $T_{e}(r, \vartheta)$ measured with the ECE-I system (outer plots) and compared to the modeled magnetic field topology as field line's connection length (middle plots, adapted from [15]). The right figure shows an example in standard $I_{P}$ and $B_{t}$ configuration while in the left figure $I_{P}$ and $B_{t}$ were reversed. The measurement position is sketched in the topology plots.

The enlarged figures on the left (reversed settings) and right (standard settings) show $\hat{T}_{e}(r, \vartheta)$ as electron temperature normalized to the phase of the discharge without perturbation field applied. In the middle the correspondent magnetic topology in the observation vicinity is shown calculated with the ATLAS simplectic field line mapping code [9, 12]. The exact location of the observation volume is depicted as yellow dashed line. This comparison shows that $\hat{T}_{e}$ is reduced to about 0.8 where short field lines dominate the measurement position. In contrast $\hat{T}_{e}$ is nearly unaffected in case long, ergodic field dominate the observation volume. They connect this region directly with regions deeper inside the plasma (typically the next innermost resonant island chain) transporting heat by parallel conduction into this observation volume. As the diffusion of these ergodic field lines is the necessary ingredient this ergodic domain is characterized by a diffusive transport. On the opposite, the short $L_{c}$ domains with reduced $\hat{T}_{e}$ values connect directly to the DED target on the HFS. Therefore they open a parallel loss channel governing this plasma vicinity by direct parallel, convective transport. The impact of these short $L_{c}$ - so called laminar domains - on the electron density $n_{e}(r, \vartheta)$ is elaborated with the He beam diagnostic. An example for these measurements is shown in the right part of figure 4. Here results of sweeping the DED currents are shown. During this sweep the imposed magnetic topology remains unchanged [13] but is evenly shifted along the poloidal angle. Therefore we get a map of $n_{e}(r, \vartheta)$ and $T_{e}(r, \vartheta)$ for $5 \mathrm{deg}<\vartheta<15 \mathrm{deg}$ from the profile measurements of the He beam diagnostic. The measurement results are shown in the upper right $\left(n_{e}(r, \vartheta)\right)$ and lower right figure $\left(T_{e}(r, \vartheta)\right)$ of figure 4, right part. The left picture in this figure shows $L_{c}(r, \vartheta)$ convoluted with the He beam divergence and therefore representing the evolution of the magnetic topology during this sweep. Here the effect discussed before is clearly obvious. In the beginning of this sweep (point 1) long, ergodic field lines determine the measurement location and $n_{e}$ as well as $T_{e}$ are reduced by $30 \%$ for $n_{e}$ and $15 \%$ only for $T_{e}$ compared to the no DED reference point. At position 2 both parameters are reduced by $50 \%$ for $n_{e}$ and $30 \%$ for $T_{e}$. This again emphasizes the convective loss imposed by the short field lines and the diffusive characteristics of the ergodic domain. This findings could be elaborated in great detail due to the diagnostic capabilities at TEXTOR 
as described for example in $[12,13,14,15,24]$. The ergodic domains represent a diffusive transport domain and they show an increase in the radial particle transport by $20 \%$ for the $m / n=12 / 4$ base mode. However, the radial heat transport is unaffected which is a finding contradictory to the classical ergodic transport hypothesis and the findings at TORE-Supra [25]. The laminar flux tubes act as scrape off layer (SOL) of the helical, multipolar, ergodic divertor structure induced. It represents a convective loss channel where strong parallel particle flux leads to a localized reduction in $n_{e}$. However, the temperature reaction does not follow and the conductive loss in this vicinity is small.

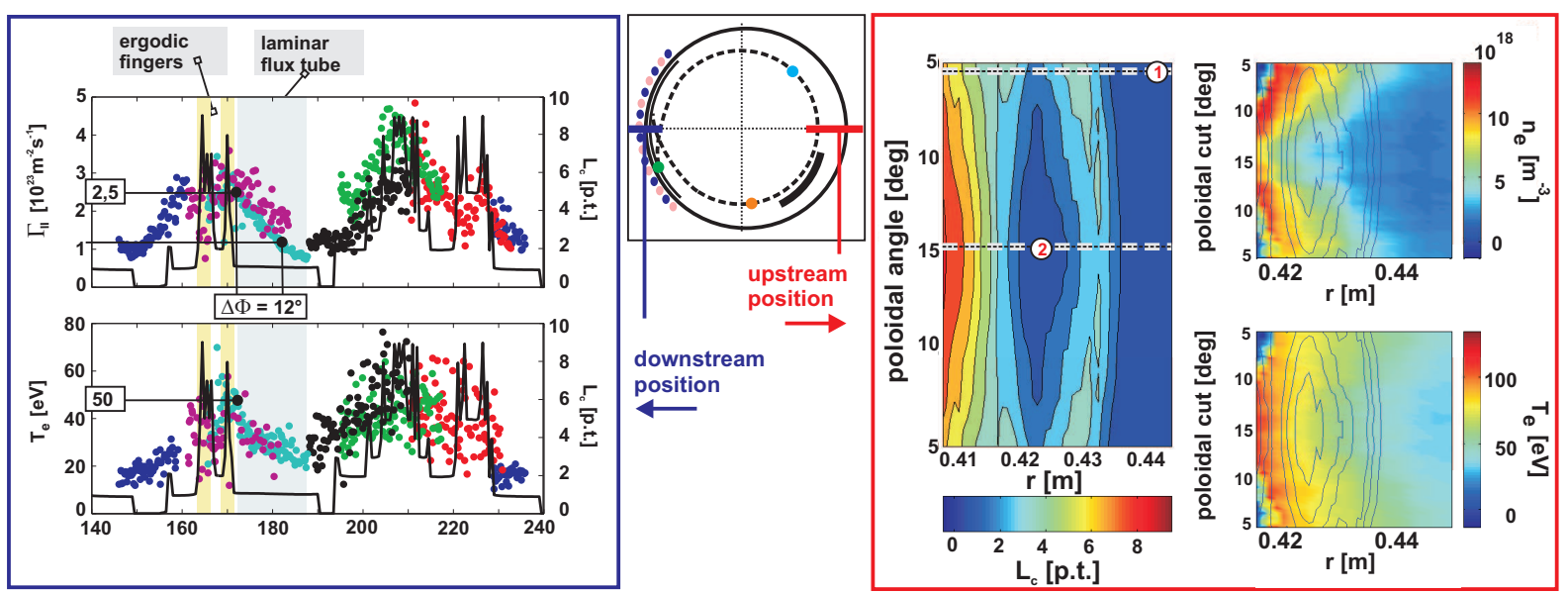

FIGURE 4. Measurements at two characteristic positions in the helical divertor structure imposed by the DED. The plot on the right shows the poloidal and radial distribution of $n_{e}(r, \vartheta)$ (upper right figure) and $T_{e}(r, \vartheta)$ (lower right figure) measured at the LFS in the vicinity of a laminar flux tube, i.e. the upstream region of the divertor structure (adapted from [15]). The picture on the left shows results from Langmuir probe measurements (particle flux $\Gamma_{\|}(\varphi)$ along toroidal angle in upper plot and $T_{e}(\varphi)$ along toroidal angle in the lower plot) on the DED target where the flux tubes end, i.e. the downstream region (adapted from [24]). The middle figures sketches a projection of the toroidal evolution of a laminar flux tube in the poloidal plane. This is the helical SOL geometry in $2 \mathrm{D}$ and the upstream and downstream diagnostic locations are indicated.

The functionality of the laminar flux tubes as SOL were elaborated in more detail combining the findings from the He beam measurements at LFS and the LP data on the DED target. The He beam is located in the upstream position of this helical divertor, on the opposite side of the divertor strike points on the DED target. Here - in the so called downstream position - the LP are located. In the sweep mode one can elaborate a toroidal profile of the particle flux as well as of $T_{e}$ on the target. The result for a comparable discharge is shown in the left part of figure 4 . The upper plot shows a toroidal profile of the parallel particle flux $\Gamma_{\|}(\varphi)$ (left ordinate) compared to the $L_{c}$ of the field lines hitting the target at these positions (right ordinate). The lower plot shows as correspondent plot a comparison of the electron temperature $T_{e}(\varphi)$ and $L_{c}(\varphi)$. The e-folding length in the laminar flux tubes can be elaborated at both measurement positions. This allows to deduce the wetted area and to conclude a resulting balance of the input heating power and the power deposited on the DED target [19]. This analysis showed that in the $m / n=12 / 4$ base mode 50\% of the absolut heat flux is deposited by the laminar flux tube and the other half by ergodic field lines. This proves the importance of the short field lines bundled into the SOL flux tubes. They imprint a poloidal modulation in the electron density and temperature fields. This means that the description of stochastic edge layers need a three dimensional description. Poloidally averaged approaches accounting only on radial profiles in the modified transport coefficients can not resolve this poloidal fine structure [15].

These examples were taken as results from static DED operation. One subsequent analysis tackled the question if these structures will follow the rotating field in the DED AC modus. In figure 5 an example from SHE measurements is shown. This diagnostic is capable to follow events on this time scale and therefore they allow an appropriate comparison to the He beam measurements discussed before. The left plot in figure 5 shows the modulation of $n_{e}(r, t)$ (left plots) and $T_{e}(r, t)$ (right plots) for a discharge with $\mathrm{AC}(+)$ rotation, i.e. the field is rotating in ion-diamagnetic drift direction (upper row) and a discharge with opposite rotation direction (lower row).

This measurement resolved for the first time that the modulation of $n_{e}$ and $T_{e}$ is apparent also with a $1 \mathrm{kHz}$ rotating magnetic field. A modulation of $40 \%$ in $n_{e}$ and of $25 \%$ in $T_{e}$ is clearly visible. The data shown are averaged over one He beam pulse and therefore the time axis is normalized to the DED period $\tau_{D E D}$. Comparing the modulation in the 
upper and the lower row a phase between the modulation of the $\mathrm{AC}(+)$ and the $\mathrm{AC}(-)$ case can be seen. To compare both discharges in more detail, a time trace at $r=0.446 \mathrm{~m}$ was extracted and compared to the modulated perturbation field $B_{r}(t)$ (green curve, right ordinate) at this position, calculated as vacuum field from the perturbation mode spectrum [8]. The $n_{e}$ and $T_{e}$ time traces were shifted such that $B_{r}(t)$ for both DED field directions became congruent. This comparison clearly exhibits a phase between both density time traces and an even larger phase between the temperature time traces. The systematic comparison of these phase shifts between $n_{e}(t)$ and $T_{e}(t)$ and relative to the vacuum magnetic perturbation field, respectively allows to gain information on the field penetration and screening. This is an important question as all examples shown here and nearly the complete analysis in the field of RMP research is done in the vacuum approximation. This means that the external perturbation field is calculated and superimposed to the unperturbed magnetic equilibrium without accounting on the plasma's feedback. This simplification may be justified in the far plasma edge but as deeper the field penetrates as easier screening current can evolve and as stronger effect of the plasma rotation on the field penetration is expected. These new and unique measurements shall contribute to the assessment of these field penetration physics in future.
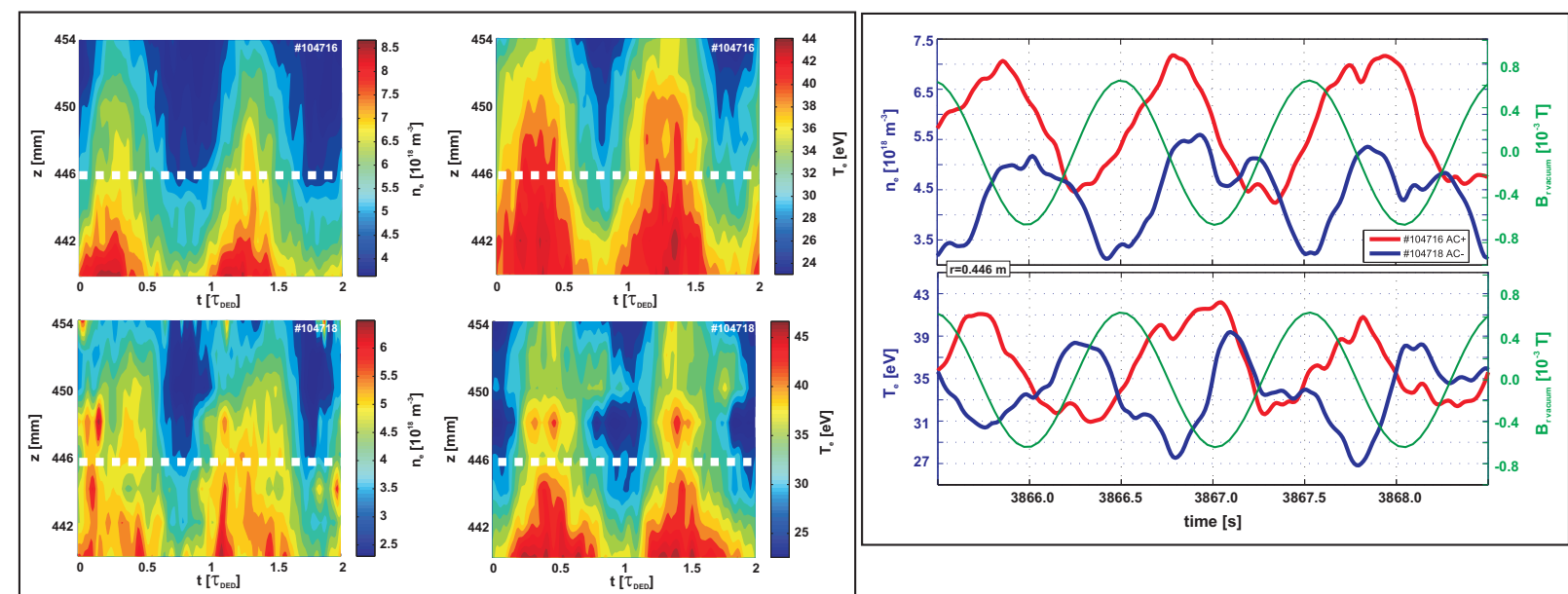

FIGURE 5. Figures on the left show the modulation of $n_{e}\left(r, \tau_{D E D}\right)$ (left plots) and $T_{e}\left(r, \tau_{D E D}\right)$ (right plots) during $1 \mathrm{kHz}$ DED-AC operation. The upper plots show results from DED rotating field in co ion diamagnetic drift direction (discharge \#104716) and the lower plots into the counter direction (\#104178). The time period of the DED rotation was used as time unit $\tau_{D E D}$. On the right the modulation of $n_{e}\left(\tau_{D E D}\right)$ (upper plot) and $T_{e}\left(\tau_{D E D}\right)$ (lower plot) during $1 \mathrm{kHz}$ DED-AC operation. These time traces were deduced at $r=0.446 \mathrm{~m}$, this radial position is sketched as white, dashed line in the figures on the left. The blue curves with ordinate on the left are for $\mathrm{AC}(+)$ rotation and the red curve with ordinate on the left are for $\mathrm{AC}(-)$ rotation. The green curve with ordinate on the right depicts the vacuum magnetic perturbation field $B_{\text {rvacuum }}(t)$ at this radial position.

These detailed investigations allowed to resolve the local transport properties and to distinguish the SOL flux tubes from the diffusive transport domain. This gave rise to further development of the exhaust control mechanisms with the stochastic boundary of TEXTOR-DED. Figure 6 shows the two particle confinement regimes which were achieved by adapting the plasma position and by that evoking different magnetic topologies. The left part of this figures show the time traces of the line averaged central electron density $n_{e} l . a .(t)$. As indicated in the upper part of this figure the plasma was shifted $\Delta R=4.0 \mathrm{~cm}$ to the inside of TEXTOR, i.e. towards the perturbation source. This leads to an improved particle confinement with an increase of $20 \%$ in $n_{e} l . a .(t)$ and with peaked density profiles [26]. This effect is a resonant effect. When we ramp up the DED current this density increase appears to be stepwise. Each step was identified to take place when the next resonant island chain is reached by the perturbation and therefore is ergodised. At this point a direct connection between the island chain's $\mathrm{x}$-points and the wall evolve - the so called heteroclinic tangles [27]. They represent a short cut between the wall and a deeper plasma region with higher $T_{e}$, changing the sheath potential on the wall and by that presumably the radial electric field. Evidence for this comes from a spin up of the poloidal rotation measured in coincidence with the stepwise density increase [26]. In contrast to this improved particle confinement a controlled degradation is found for plasmas shifted $\Delta R=2 \mathrm{~cm}$ to the outside, i.e. away from the perturbation source. Here the so called particle pump out (PO) effect steps out. This is a common phenomena in RMP ELM control experiments [2,3] and the elaboration of the underlying mechanisms is of great importance. One candidate for the particle PO observed at TEXTOR-DED for the settings described is that we lower the impact of the 
DED nearfield (laminar flux tubes) in the magnetic topology enhancing the ergodic character of the perturbed field lines. This shall lead to an overall enhanced radial particle transport as seen in the localized measurements before and therefore a particle loss channel which is not balanced by external fueling is opened resulting in the particle PO.

The right part of figure 6 shows an example for a beneficial effect discovered in direct correlation to the occurrence of the particle PO. Two discharges - both with DED perturbation field applied - are compared and the $n_{e} l . a .(t)$ time traces (blue and red curves with ordinate on the left) are shown as well as the carbon density in the plasma centre (black and green curve with ordinate on the right). This measurement was deduced from charge exchange spectroscopy on the neutral beam heating. In discharge \#103902 the DED current $I_{D E D}=1.4 \mathrm{kA}$ was set to a moderate level and no particle PO was evoked. In contrast, when we increased the DED current to $I_{D E D}=2.8 \mathrm{kA}$ we observed an particle PO of $22 \%$ and contemporary a reduction in the carbon density (and concentration also) of $30 \%$. This proves that correlated to the particle PO effect an screening of the intrinsic carbon was seen [28].
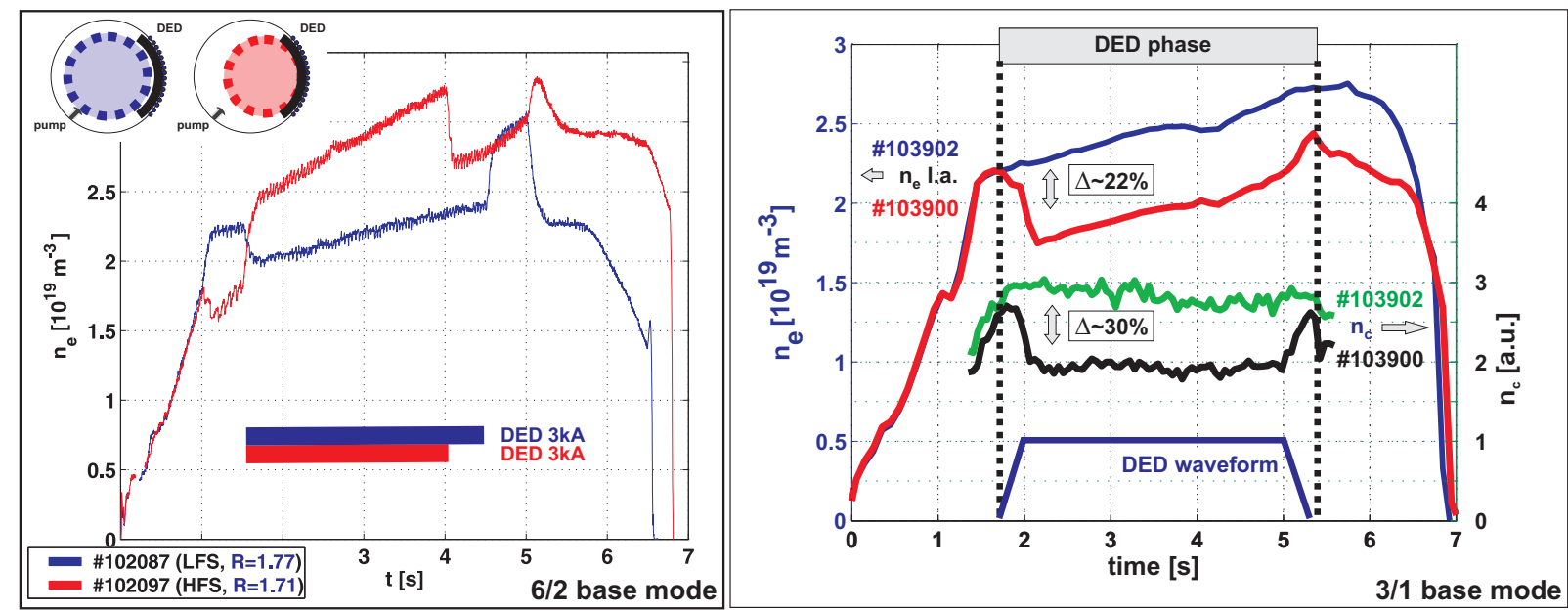

FIGURE 6. Control of the particle confinement properties by the DED imposed stochastic magnetic field. The figure on the left shows examples for the two regimes obtained by adapting the plasma position. Blue curve shows the central line averaged density $n_{e} l . a .(t)$ for a discharge moved $\Delta r \approx 2.0 \mathrm{~cm}$ towards the LFS, the red curve shows $n_{e} l . a .(t)$ for a comparable discharge but with a displacement of $\Delta r \approx 4.0 \mathrm{~cm}$ towards the HFS, i.e. towards the perturbation source. A pronounced density increase showed up for the latter configuration while in the first one the so called particle pump out was evoked. The figure on the right shows the correlation of the occurrence of this controled degradation of the particle confinement $\left(n_{e} l . a .(t)\right.$ for a discharge with moderate (blue curve, left ordinate) and high DED current (red curve with left ordinate)) and an 30\% screening of the intrinsic carbon (low current discharge in green with ordinate on the right and high current case correspondingly as black curve.).

\section{CONCLUSION}

With the results shown exemplarily in this paper the identification of the basic transport characteristics in the helical, multipolar, ergodic divertor structure imposed by the DED was demonstrated. The studies related to this topic and published in several papers (see for example [24, 29, 23, 12, 30, 8, 31, 13, 15, 26, 14]) revealed the basic transport mechanisms in close correlation to the modeled magnetic topology. These investigations gave rise to more detailed analysis of the generic transport processes in the stochastic boundary of TEXTOR-DED. A large effort was dedicated to the resolution of turbulence properties. Here as outstanding result a reduction of the turbulence level with application of the DED perturbation field was found and a reversal of the radial turbulent particle flow in the ergodic regions $[32,33]$. These results were obtained with the fast reciprocating probe system. A new scanning reflectometer allows to resolve characteristics of zonal flows and deducing the poloidal rotation velocity. Herewith a change in the radial electric field was observed caused by the increasing field line diffusion with high perturbation field [34]. The poloidal rotation accordingly spins up into ion-diamagnetic drift direction. These findings were confirmed by measurements with the hydrogen diagnostic beam RUDI [35]. Also the application of the upgraded reflectometer system allowed to observe geodesic acoustic modes in the plasma edge and resolve their role for density fluctuations and the impact of RMP on this important high frequency zonal flow domain [36].

The heat transport characteristics in the transition region between the stochastic layer and the core plasma, i.e. the 
non destroyed magnetic island chains (see figure 1, right part as example) was investigated with the ECE-I system also. The DED can produce large $2 / 1$ magnetic islands evoked by a $2 / 1$ neoclassical tearing mode [37]. Injecting modulated ECRH heating inside the $q=2$ surface allowed to study the heat pulse propagation around these large islands with the ECE-I system. Here a large phase delay was observed for the heat pulses traveling towards the island O-point compared to heat pulses traveling through the X-point region. This shows that the islands evoke a local barrier for the radial heat transport which is redirected through the island's X-points [38, 39]. A very good example for combined application of several advanced diagnostic systems for analysis of this radial domain is reported in reference [40]. Here the reflectometer system, the ECE-I system and the upgraded soft X-ray diagnostic were used to detect and analyze secondary structures aside the large 2/1 NTM islands and their harmful impact on the plasma confinement was resolved.

In conclusion the examples discussed in this paper and the ones outlined in the conclusion show that the advanced edge diagnostic equipment at the TEXTOR tokamak in combination with the DED as uniquely flexible source of RMP allow to resolve basic and generic transport processes. These investigations contribute to a broad field of topics in the research on field penetration, structural formation and the transport processes evoked by RMP.

\section{REFERENCES}

1. Evans T.E. et al., Nature of Physics 2, 419-423 (2006).

2. Fenstermacher M. et al., Journal of Nucl. Mater. 363-365, 476-483 (2007).

3. Liang Y. et. al., Phys. Rev. Lett. 98, 265004 (2007).

4. König R. et al., Plasma Physics and Controlled Fusion 44, 2365-2422 (2002).

5. Morisaki T. et al., Journal of Nucl. Mater. 337-339, 154-160 (2005).

6. Masuzaki S. et al., Nuclear Fusion 42, 750-758 (2002).

7. Finken K.H. (Editor), Report des FZJ, Jül-3285, 1996.

8. Finken K.H. et al., Schriften des Forschungszentrums Jülich, Band 45, ISBN 3-89336-418-8, 2005.

9. Abdullaev SS. et al., Nuclear Fusion 43, 299-313 (2003).

10. Eich T. et al., Nuclear Fusion 40, 1757-1772 (2000).

11. Kobayashi M. et al., Nuclear Fusion 44, S64-S73 (2004).

12. Jakubowski M.W. et al., Nuclear Fusion 44, S1-S11 (2004).

13. Schmitz O., D. Harting et al., Journal of Nucl. Mater. 363-365, 680-685 (2007).

14. Jakubowski M.W., O. Schmitz et al., Phys. Rev. Lett. (????).

15. Schmitz O. et al., Nuclear Fusion in press (2007).

16. Schweer B. et al., Journ. Nucl. Mater. 266-269, 673 (1999).

17. Pospieszczyk A. et al., Nucl. Inst. and Meth. in Phys. Research B72, 207-223 (1992).

18. Brix M., PhD thesis, Universität Bochum, D294, Report des FZJ, Jül-3638, 1998.

19. O. Schmitz, PhD thesis, Universität Düsseldorf, Report des FZJ, Jül-3285, 2006.

20. Kruezi U., Schriften des Forschungszentrums Jülich, Band 62, ISBN 978-3-89336-476-3, 2007.

21. Classen I. et al., Europhysics conference abstracts 29C (2005).

22. Park H. et al., Rev. Sci. Instrum. 74, 4239 (2003).

23. Lehnen M. et al., Journal of Nucl. Mater. 363-365, 377-381 (2007).

24. Lehnen M. et al., Plasma Physics and Controlled Fusion 47, B237-B248 (2005).

25. Ghendrih Ph. et al., Nuclear Fusion 42, 1221-1250 (2002).

26. Finken K. H. et. al., Phys. Rev. Let. 98, 065001 (2007).

27. Jakubowski M. et al., Journal of Nucl. Mater. 11111, 11111 (2007).

28. Telesca G. and Schmitz O. et al., Europhysics conference abstracts (2007).

29. Lehnen M. et al., Journal of Nucl. Mater. 337-339, 171-175 (2005).

30. Jakubowski M.W. et al., AIP conference proceedings CP812, 195-198 (2006).

31. Schmitz O. et al., Europhysics conference abstracts 29C (2005).

32. Xu Y. et. al., Plasma Phys. and Contr. Fusion 47, 1841-1855 (2005).

33. Xu Y. et. al., Phys. Rev. Let. 97, 165003 (2006).

34. Kraemer-Flecken A. et. al., Nucl. Fusion 46, S730-S742 (2006).

35. Unterberg B. et al., Journal of Nucl. Mater. 11111, 11111 (2007).

36. Kraemer-Flecken A. et. al., Phys. Rev. Let. 97, 045006 (2006).

37. Koslowski H. R. et. al., Plasma Physics and Controlled Fusion 48, B53-B61 (2006).

38. Spakman G.W. et al., Europhysics conference abstracts (2007).

39. Classen I. et. al., Phys. Rev. Lett. 98, 035001 (2007).

40. Liang Y. et. al., Nuclear Fusion 47, L21-L25 (2007). 


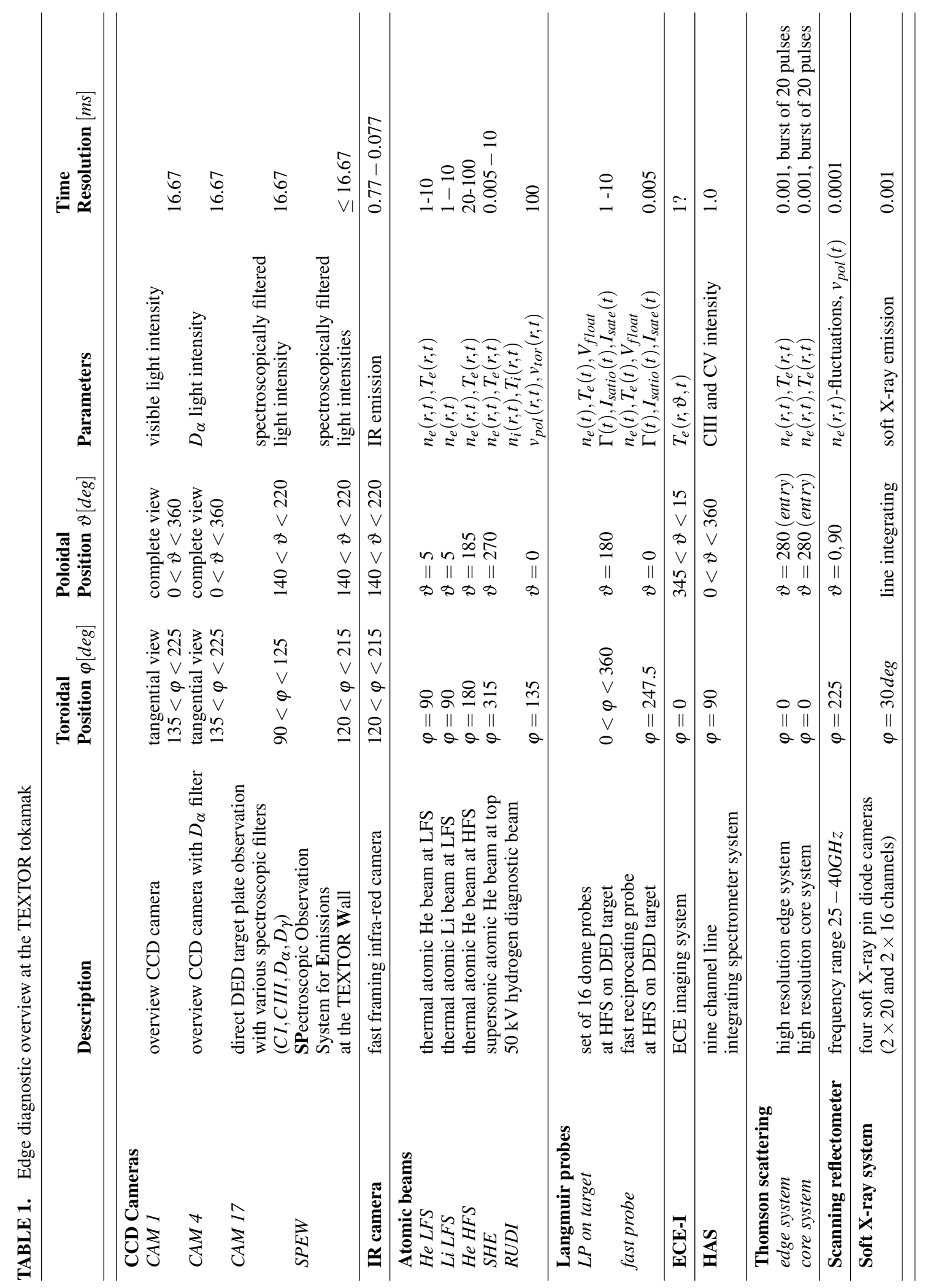

